\title{
Designing Physical Topologies that Enable Survivable Routing of Logical Rings
}

\author{
Aradhana Narula-Tam ${ }^{1}$, Member, IEEE, Eytan Modiano ${ }^{2}$, Senior Member, IEEE \\ ${ }^{1}$ MIT Lincoln Laboratory, 244 Wood Street, Lexington, MA 02420 \\ ${ }^{2}$ MIT Laboratory for Information and Decision Systems, Cambridge, MA 02139 \\ Email:
}

\begin{abstract}
In a WDM-based network, a single physical link failure may correspond to multiple logical link failures. As a result, 2-connected logical topologies such as rings routed on a WDM physical topology, may become disconnected after a single physical link failure. We consider the design of physical topologies that ensure logical rings can be embedded in a survivable manner. First, we develop necessary conditions for the physical topology to be able to embed all logical rings in a survivable manner. We then use these conditions to provide tight bounds on the number of physical links that an $N$ node physical topology must have in order to support all logical rings of size $K$. We show that when $K \geq 4$ the physical topology must have at least $4 N / 3$ links, when $K \geq 6$ it must have $3 N / 2$ links, and when $K \geq N \square 2$, it must have at least $2 N \square 4$ links. We design a topology that meets the above bounds for $K=N \square 2$ using a dual-hub architecture. Finally, we observe, through simulation experiments, that designing the physical topology for supporting all logical rings in a survivable manner does not use significantly more physical links than a design that only supports a small number of logical rings. Hence, our approach of designing physical topologies that can be used to embed all possible ring logical topologies does not lead to a significant over-design of the physical topology.
\end{abstract}

\section{Introduction}

Wavelength Division Multiplexing (WDM) based networks consist of a logical topology defined by a set of nodes and lightpaths connecting the nodes and a physical topology defined by the set of nodes and the fiber connecting them. Although both the logical and physical topologies may be independently tolerant to single link failures, once the logical topology is embedded on the physical topology, the logical topology may no longer be survivable to single physical link failures. Each physical fiber link may carry multiple lightpaths. Hence, the failure of a single physical link, can lead to the failure of multiple links in the logical topology that may subsequently leave the logical topology disconnected.

As a simple illustrative example, consider the logical and physical topologies shown in Figure 1. The logical topology is a ring with nodes ordered 1-3-4-5-2-1. Clearly, such a ring topology is 2 -connected, and would remain

The work of A. Narula-Tam is sponsored by the United States Air Force under contract number F19628-00-C-0002. The work of E. Modiano is sponsored by DARPA under contract number MDA972-02-1-0021. Opinions, interpretations, recommendations and conclusions are those of the author and are not necessarily endorsed by the United States Government. connected if one of its links failed. The five logical links of this ring can be routed on the physical topology as shown in Figure 1a, where each physical link is labeled with the logical link that traverses it. For example logical link $(1,3)$ traverses physical links $(1,5)$ and $(5,3)$. As can be seen from the figure, no physical link supports more than one logical link. Hence, the logical ring would remain protected even in the event of a physical link failure.

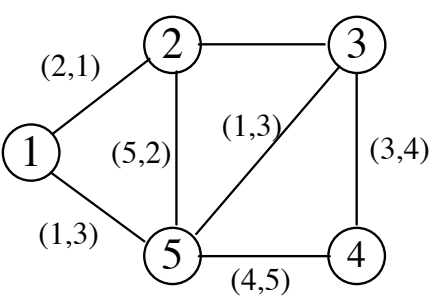

a) Physical topology

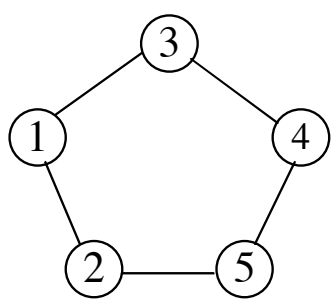

b) Logical topology
Figure 1: Survivable routing of a ring logical topology.

Alternatively, had we routed logical link $(1,3)$ on physical links $(1,2)$ and $(2,3)$ the routing would no longer be survivable because physical link $(1,2)$ would have to support both logical links $(1,3)$ and $(2,1)$ hence its failure would leave the logical topology disconnected. Furthermore, for many logical topologies, no survivable routings can be found. For example, if the logical topology was a ring with nodes ordered 1-4-2-3-5-1 then it can be easily seen that no routing exists that can withstand a physical link failure. Hence, it is clear that although the logical topology of the network may be connected, once it is embedded on top of a WDM physical network, it may no longer withstand a physical link failure (e.g., fiber cut).

Our focus is on the design of physical topologies capable of supporting ring logical topologies in a survivable manner. While there has been a great deal of work in the area of optical layer protection [1-8], this survivable routing formulation is a new approach to network protection that has significant implications on the design of future WDM-based networks. Most previous work in WDM network protection has focused on restoration mechanisms that restore all lightpaths in the event of a physical link failure. Link based restoration recovers from a link failure by restoring the failed physical link, hence simultaneously restoring all of the associated lightpaths $[1,2,5]$. This is often done using optical loop-back protection $[1,2,4]$. In contrast, path based protection restores each of the lightpaths independently, by finding an 
alternative end-to-end path for each lightpath [1,2,7]. In many cases it is indeed necessary to restore all failed lightpaths. However, in other cases some level of protection is provided in the electronic layer and restoration at the physical layer may not be necessary. For example, when the electronic layer consists of SONET rings, single link failures can be recovered through loopback protection at the electronic layer. In this case, providing protection at both the optical and electronic layers is somewhat redundant. Another less obvious example is that of packet traffic in the internet where multiple electronic layer paths exist between the source and destination and the internet protocol (IP) automatically recovers from link failures by rerouting packets.

In such cases, a less stringent requirement may be imposed on the network - for example that the network remain connected in the event of a physical link failure. This approach, of course, is not suitable for all situations. For example, when a network is carrying high priority traffic with Quality of Service and protection guarantees, it may still be necessary to provide full restoration. However, when a network is used to support best effort internet traffic, guaranteeing connectivity may suffice.

In $[9,10]$ we considered the problem of embedding ring logical topologies on a given physical topology in a manner that ensures the logical topology remains connected in the event of a physical link failure. We call such embeddings "survivable". One of the key results observed in [10] is that for many physical topologies it is not possible to embed ring logical topologies in a survivable manner. For example, almost $50 \%$ of 9 node rings cannot be embedded, in a survivable manner, in the 11 node NJLATA network [10]. Similar results were also obtained for other commonly used physical topologies. Hence, in this paper we focus on the dual problem: How should the physical topology be designed so that it can support logical rings in a survivable manner? In particular, we investigate properties of physical topologies that enable multiple logical rings to be embedded in a survivable manner and use these properties to design suitable physical topologies. Such design is particularly useful for service providers that design their network infrastructure in order to serve customer requests for lightpath connections.

Since at present most protected logical topologies tend to be rings, in this paper we focus on the design of physical topologies for supporting logical rings in a survivable manner. Alternatively, the service provider can provide restoration at the physical layer. However, such restoration may duplicate functionality provided at higher layers and be wasteful of resources. Also, the physical layer restoration must be fast enough to be compatible with the requirements of the higher layer (e.g., must restore the fiber cut before SONET initiates loopback protection). Our approach to the physical topology design allows a service provider to route the lightpaths that constitute the logical ring along completely disjoint paths, thereby preserving the connectivity properties of the ring.

We consider the design of $N$ node physical topologies that can support survivable routings of ring logical topologies of size $K \sqcap N$. Since rings of size 3 can be trivially embedded in a survivable manner on any 2connected physical topology, we focus on the problem of embedding rings of size $K \geq 4$. We approach the design problem from two angles. First we develop necessary conditions on the physical topology for ensuring all $K$ node ring permutations can be embedded in a survivable manner. These conditions lead to lower bound requirements on the number of physical links. Second, we formulate the problem as an Integer Linear Program (ILP) to design the physical topology using the minimum number of physical links while allowing a set of random logical topologies to be embedded in a survivable manner. Finally we use the insights gained from solving the ILP and our analytical results to design physical topologies which can support all ring permutations in a survivable manner using the minimum number of physical links.

\section{Conditions for survivable routing}

We consider a bi-directional physical topology with nodes $N$ and edges $E$. Similarly, each bi-directional logical topology consists of a set of nodes $N_{L}$ and edges $E_{L}$.

A cut is a partition of the set of nodes $N$ into to subsets $S$ and $N \square S$. The cut-set corresponds to the set of edges in $E$ that have one endpoint in $S$ and the other in $N \square S$. In [9] it was shown that a necessary and sufficient condition for survivable routing of a logical topology is that no single physical link is shared by all logical links belonging to a cut-set of the logical topology. In other words, not all of the logical links belonging to a cut-set can be routed on the same physical link. This condition must hold for all cutsets of the logical topology. For ring logical topologies, this implies that no two logical links can be mapped on the same physical link.

Furthermore in [13] we develop some necessary conditions on the physical topology to ensure survivable routing of ring logical topologies. Consider any random ring logical topology. For any cut $\langle S, N \square S\rangle$ of the physical topology, let $\left|\mathrm{CS}_{\mathrm{P}}(S, N \square S)\right|$ be the number of physical links along this cut and $\left|\mathrm{CS}_{\mathrm{L}}(S, N \square S)\right|$ be the number of logical links traversing the same cut. Clearly, in order to be able to route the logical links along disjoint physical paths, $\left|\mathrm{CS}_{\mathrm{P}}(S, N \square S)\right|$ must be greater than or equal to $\left|\mathrm{CS}_{\mathrm{L}}(S, N \square S)\right|$ for each cut. This condition is necessary, but not sufficient to insure that a survivable routing exists for a particular ring logical topology. For embedding all possible $K$ node ring logical topologies in a survivable manner we obtain the following necessary condition on the physical topology. 
Theorem 1 An N node physical topology can support any possible $K$ node ring logical topology in a survivable manner only if for any cut of the physical topology $\langle S, N \square S\rangle,\left|C S_{P}(S, N \square S)\right| \geq 2 \min (|S|,|N \square S|, \llbracket K / 2 \square$.

Proof: see [13]. The proof is by construction showing that there exists a ring logical topology that requires $2(\min (|S|,|N \backslash S|),[K / 2[]$ physical links along the cut.

Theorem 1 says that for all cuts of the physical topology, the number of physical links in the cut-set must be greater than or equal to twice the minimum of the number of nodes on the smaller side of the cut and $\square K / 2[$, where $\square K / 2[$ corresponds to the maximum number of nodes in a $K$ node ring logical topology that can be on both sides of the cut. Theorem 1 is a necessary but not sufficient condition. Using Theorem 1, we develop lower bounds on the number of physical links needed to embed rings of size $K=4,6$, and 8. Similar procedures can be used to develop lower bounds for larger values of $K$. Most importantly we show in Theorem 5, that to embed rings of size $K=N \square 2$, a minimum of $2 N \square 4$ physical links are needed. A summary of these lower bound results is given in Table 1. In Section $\mathrm{IV}$, these lower bounds are used to design physical topologies that can support rings of size $K$ with a minimal number of physical links.

\begin{tabular}{c|c|c}
\hline $\begin{array}{c}\text { Logical Ring } \\
\text { Size }\end{array}$ & $\begin{array}{c}\text { Physical link } \\
\text { requirement }\end{array}$ & Result \\
\hline$K=4$ & $4 N / 3$ & Theorem 2 \\
$K=6$ & $3 N / 2$ & Theorem 3 \\
$K=8$ & $1.5 \overline{5} N$ & Theorem 4 \\
$K=N \square 2$ & $2 N \square 4$ & Theorem 5 \\
\hline
\end{tabular}

Table 1: Lower bounds on the number of physical links.

We begin by developing the following lower bound on the number of physical links needed to support rings of size 4 on an $N$ node physical topology.

Theorem 2: To support all logical rings of size $K \geq 4$, an $N$ node physical topology must have at least $4 N / 3$ links.

To prove Theorem 2, we utilize the following lemma which is obtained by applying Theorem 1 to two node cutsets of the physical topology.

Lemma 1: Any node of degree 2 must have physical links to nodes of degree 4 or higher.

Proof: Suppose a node of degree 2 has a physical link to a node of degree 3 , then the cut-set consisting of the degree 2 node and its degree 3 neighbor contains only 3 links. However, since the cut-set contains two nodes, Theorem 1 requires a minimum of 4 cut-set links.

Proof of Theorem 2: Let $d_{i}$ be the number of nodes with degree $i$ in the physical topology. Then the number of links in the physical topology, $L$, is

$$
L=\prod_{i=2}^{N \square 1} \frac{i d_{i}}{2}=d_{2}+\frac{3 d_{3}}{2}+\square_{i=4}^{N \square 1} \frac{i d_{i}}{2} .
$$

From Lemma 1, we know that every degree 2 node must have physical links to two nodes which have degree 4 or higher, therefore

$$
d_{2} \square \bigsqcup_{i=4}^{N \bigsqcup 1} \frac{i}{2} d_{i} .
$$

Substituting Eq. (2) into Eq. (1) yields

$$
L \geq 2 d_{2}+\frac{3}{2} d_{3} \text {. }
$$

Since nodes of degree $i$ add a minimum of $i / 2$ physical links to the physical topology,

$$
L \geq \frac{2 d_{2}+3 d_{3}+4\left(N \square d_{2} \square d_{3}\right)}{2}=2 N \square d_{2} \square \frac{d_{3}}{2} .
$$

Equations (3) and (4) require that the number of physical links must be

$$
L \geq \max \left(2 d_{2}+\frac{3}{2} d_{3}, 2 N \square d_{2} \square \frac{d_{3}}{2}\right) .
$$

We can determine the values of $d_{2}$ and $d_{3}$ that minimize the number of physical links required. The minimum value occurs when $2 d_{2}+\frac{3}{2} d_{3}=2 N \square d_{2} \square \frac{d_{3}}{2}$, i.e., when $d_{2}=\frac{2 N \square 2 d_{3}}{3}$. Substituting this value of $d_{2}$ into Equation (1) yields

$$
L \geq \frac{4 N}{3}+\frac{d_{3}}{6} \geq \frac{4 N}{3} .
$$

Next we develop a lower bound on the number of physical links required to support rings of size $K=6$ and $K=8$.

Theorem 3: To support all logical rings of size $K \geq 6$, an $N$ node physical topology must have at least $3 N / 2$ links.

Theorem 4: To support all logical rings of size $K \geq 8$, an $N$ node physical topology must have at least $1.5 \overline{5} N$ links.

The proofs of Theorem 3 and 4 follow very similar lines to that of Theorem 2 and are given in the appendix.

The above approach can be pursued further to obtain bounds on the number of physical links required to support logical rings of sizes greater than 8 . However, such proofs become increasingly complex for larger values of $K$. Instead, the following Theorem provides a lower bound on the number of physical links needed to embed rings of size $\mathrm{K}=\mathrm{N}-2$ or larger.

Theorem 5: The minimum number of physical links necessary to support all logical rings of size $K \geq N \square 2$ (for $K \geq 4)$ in a survivable manner is $2(N \square 2)$.

To prove Theorem 5 we show that for any physical topology with fewer than $2(N \square 2)$ links, we can find an $N \square 2$ node ring logical topology where each logical link requires two physical links (for a total of 2(N口2) links). 
Hence a physical topology with fewer than 2(N口2) links cannot support all $N \square 2$ node logical topologies. The proof of Theorem 5 is given in the Appendix. The above theorem provides a necessary condition on the number of physical links required to embed all logical rings of size $N \square 2$ or greater. Later, in Section IV, we will show that this number is also sufficient for $N$ even. We will also show that when $N$ is odd $2 N \square 3$ links are sufficient to embed all rings of size less than or equal to $N$.

The above Theorems and Lemmas provide us with lower bounds on the number of physical links that the physical topology requires. They also give us some insights regarding the structure of the topology (e.g., low degree nodes must be connected to high degree nodes, etc.). However, they do not directly provide us with a physical topology design. In order to obtain additional insight, we next apply Integer Linear Programming techniques to design physical topologies. In the last section we will use these insights to design physical topologies that meet the above bounds.

\section{ILP Formulation}

In this section we develop an Integer Linear Program (ILP) formulation for designing physical topologies that can support large numbers of logical rings in a survivable manner. We consider the problem of finding a physical topology with a minimum number of physical links and the associated survivable routings for a batch of $R$ ring logical topologies with $K$ nodes each. We use the ILP below to determine a survivable routing for each of the $R$ rings, which ensures that each logical topology remains connected even in the event of a physical link failure. In order to route a logical link $(s, t)$ on the physical topology one must find a corresponding path on the physical topology between nodes $s$ and $t$. Such a lightpath consists of a set of physical links connecting nodes $s$ and $t$ as well as wavelengths along those links. Let $f_{i j}^{s t}=1$ if logical link $(s, t)$ is routed on physical link $(i, j)$ and 0 otherwise.

Our linear programming algorithm starts with a fullyconnected physical topology and assigns a cost of 1 to each physical link that is used. The batch of $R$ rings is embedded simultaneously, and we assign $y_{i j}=1$ if any lightpath uses physical link $(i, j)$. We can now formulate the physical topology design problem as the following ILP, with the objective of minimizing the total number of physical links used.

Minimize $\square_{(i, j) \square E} y_{i j}$ subject to:

a) Connectivity constraints: for each pair $(s, t)$ in each

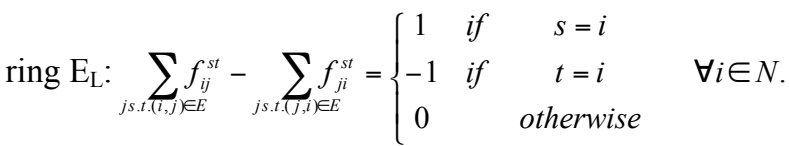

b) Survivability constraints for each logical ring $E_{\mathrm{L}}$ : $\square_{(s, t) \square E_{L}} f_{i j}^{s t}+\square_{(s, t) \square E_{L}} f_{j i}^{s t} \square 1, \quad \square(i, j) \square E$.

c) Physical link use constraints: $y_{i j} \geq f_{i j}^{s t}, \quad \square(i, j) \square E, \quad \square(s, t) \square E_{L}, \square E_{L}$

d) Integer flow constraints: $f_{i j}^{s t} \square\{0,1\}$

The survivability constraint above (b) ensures that no two logical links of a ring share a physical link.

\section{A. Exact solution for ILP}

We implemented this ILP using the CPLEX software package. CPLEX uses branch and bound techniques for solving ILPs and is capable of solving ILPs consisting of up to one million variables and constraints. We have found that the solution of the ILP can only be determined for small problem instances. For example, with $N=10, K=6$, and $R=20$ rings, a physical topology solution is found within 24 hours on a SPARC Ultra 10. The resulting physical topology is shown in Figure 2. Due to the complexity of the ILP, this approach cannot be used for general design. However, attributes of the resulting topologies found through experiments provide insights to the physical topology design problem. For example, we find that (1) the physical topology does not contain a Hamiltonian cycle and (2) the physical topology has a multi-hub structure. In Section IV we use these insights to design physical topologies that are capable of embedding all rings in a survivable manner. Our designs are consistent with observations (1) and (2) and in particular we use a hub architecture.

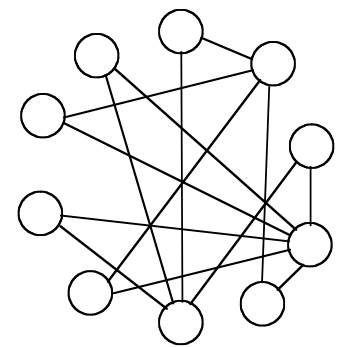

Figure 2: Topology generated by ILP for $K=6, R=20$.

\section{B. Heuristics for solving the ILP}

We examined a number of approaches for solving the above ILP with very little success. These included attempting to bundle the physical link constraints (c) and attempting to relax the integer constraints. Another approach for obtaining a feasible (but not optimal) solution is to embed the rings sequentially rather than as a batch. Expectedly, designing physical topologies by sequentially embedding rings is not nearly as efficient as embedding the rings as a batch. For example, if we embed $N$ node rings on an $N$ node topology sequentially, the first set of links will be a Hamiltonian cycle that corresponds to the first logical ring. As mentioned above, the most efficient physical topologies may not contain a Hamiltonian cycle.

One approach that did prove rather successful was to embed a small batch of about 20 rings for which the above 
ILP can be solved and then use the resulting physical topology to sequentially embed additional rings, adding physical links when necessary. The intuition behind this approach is that by embedding the small batch we avoid the negative effect that results from the sequential embedding of the first few rings. Moreover, it is reasonable to expect that the physical topology that results from embedding even a small batch is relatively close to the optimal topology for larger batches.

Hence, we combined the two approaches above, using the ILP to embed a small batch of rings in order to obtain an initial physical topology and then using this physical topology to embed more logical rings sequentially, adding physical links as required. Of course designing a physical topology in this manner that can provably support all rings requires embedding $(N \square 1)$ ! rings which is impractical for large $N$, even if the design is done off-line. However, the primary goal of this heuristic is to help obtain insight on the architecture of a physical topology that is sufficient for supporting all rings of size $N$. For $N=9$, embedding a batch of ten logical 9 node rings led to the dual hub architecture with an extra link between the hub nodes. This architecture uses 15 links and can support all 9 node rings. We will show in the next section that this dual hub design with $\mathrm{N}$ nodes can support all $\mathrm{N}$ node rings (for all $\mathrm{N}$ odd).

\section{Physical Topology designs}

The analytical and simulation (ILP) results provide valuable insights in designing physical topologies that can support ring permutations of various sizes. From Lemma 1, we know that all degree 2 nodes must have neighbors of degree 4 or higher. The physical topologies designed through the ILP simulations all have hub structures, i.e., a small number of nodes with large degree and the remaining nodes with small degree. These insights are used to design the following physical topology which can support all ring permutations of size $K \square N \square 2$.

Dual Hub Architecture: Consider a physical topology with $N$ nodes, two of which are hub nodes. Each non-hub node has degree 2 and is connected to both hub nodes. The hub nodes each have degree $N \square 2$.

An example of the resulting physical topology for $N=10$ nodes is given in figure 3 . The dual hub architecture contains $2(N \square 2)$ bi-directional physical links, the minimum required by Theorem 5 for supporting all $N \square 2$ node rings. The dual hub architecture of figure 3 can support survivable routings of all logical rings of size $K \square N \square 2$ (for $N$ even). To show this we examine the cases where the logical ring contains 0,1 , or 2 , of the hub nodes. First consider the case where the ring logical topology contains none of the hub nodes. Starting with any node in the logical ring, a path can be found to the next node via one of the hub nodes. The path to the subsequent node will then go through the other hub node. Thus consecutive lightpaths in the logical ring alternate between using each of the two hubs as intermediate nodes. Each hub node can be used as an intermediate node $(N \square 2) / 2$ times, thus we can support $N \square 2$ lightpaths.

Proofs for the cases where the logical topology contains one or both hub nodes are similar.

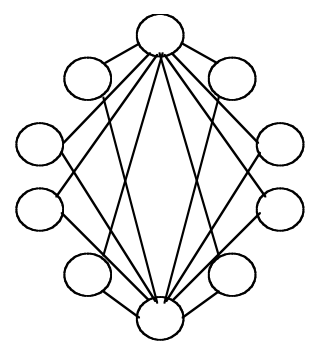

Figure 3: Topology that supports all $N \square 2$ node rings.

For $N$ even, the dual hub architecture of Figure 3 can support all size $N \square 1$ rings as well. For $N$ odd, adding an additional physical link connecting the two hub nodes allows all rings of size less than or equal to $N$ to be embedded. Furthermore, when $\mathrm{N}$ is even it can be shown that adding two diversely routed physical links to the dual hub architecture can support all rings of size $\mathrm{N}$ in a survivable manner (using a total of $2 \mathrm{~N}-2$ links).

Shown in figure 4 is a physical topology for embedding rings of size $K=4 \quad(\mathrm{~N}=12)$. In this physical topology there are only nodes of degree 2 and degree 4 . All nodes of degree 2 are connected to two nodes of degree 4 and all nodes of degree 4 are connected to four nodes of degree 2 . Thus, there are twice as many degree 4 nodes as degree 2 nodes. This example can be generalized to all values of $\mathrm{N}$ divisible by $3(\mathrm{~N} \geq 6)$. It can be easily shown that the resulting physical topology can support all rings of size $K=4$. Since the above topology contains twice as many degree 2 nodes as degree 4 nodes, clearly, it contains $4 N / 3$ physical links. By Theorem 2, this is the minimum required to support all rings of size 4 .

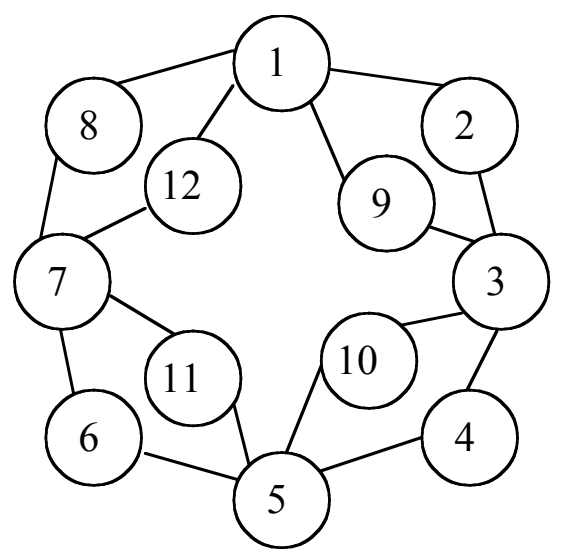

Figure 4: Physical topology for $K=4$. 


\section{Conclusion}

We consider the problem of physical topology design for embedding logical rings in a survivable manner. This problem is particularly important for service providers that design their fiber infrastructure in order to support future customer requests for lightpath connections. Since rings are a very commonly used logical topology (due to their ability to recover from failures), we focused in this paper on design for ring logical topologies. Of course, a natural extension of this work is general design for arbitrary (2connected) logical topologies.

We obtained some basic necessary conditions on the physical topology in order to be able to route logical rings in a survivable manner. We also developed tight lower bounds on the number of links that the physical topology must contain in order to be able to support all possible logical rings of size $K$ (for various values of $K$ ). Finally, we designed a number of basic physical topologies that meet these bounds. One interesting result of our work is that these physical topologies tend to have a multi-hub architecture. An important area of future work is to consider designs that do not use a hub architecture.

Finally, one may question our desire to support all $K$ node logical rings in a survivable manner. The question arises of whether we are over provisioning the physical network in our quest to support all logical rings. Notice, however, that our designs use fewer than $2 N$ physical links to support all logical rings of size $N$ or smaller. An arbitrary $N$ node logical topology requires a minimum of $N$ physical links in order to be 2-connected. Furthermore, our experiments show that attempting to embed just a small number of logical rings requires very close to $2 N$ physical links; hence, requiring the design to support all possible logical rings in fact does not result in a significant number of additional physical links. Moreover the ability to support all logical rings is useful because it allows the logical ring topology to be reconfigured. Such reconfiguration has been shown to reduce network traffic loads[12,13].

\section{Appendix: Proofs of Theorems}

Before proceeding to the proof of Theorem 3, we must first give the following useful Lemma.

Lemma 2: A node of degree 4 or degree 5 can have a physical link to at most one node of degree 2.

Proof: Suppose two nodes of degree 2 have physical links to the same node of degree 5 . Then the cut set consisting of the two degree 2 nodes and the degree 5 node contains only five links, which is less than the 6 links required by Theorem 1 .

Proof of Theorem 3: Let $d_{i}$ be the number of nodes with degree $i$ in the physical topology. Then the number of links in the physical topology, $L$, is

$$
L=\prod_{i=2}^{N \square 1} \frac{i d_{i}}{2}=d_{2}+\frac{3 d_{3}}{2}+2 d_{4}+\frac{5 d_{5}}{2}+\prod_{i=6}^{N \square 1} \frac{i d_{i}}{2} .
$$

From Lemma 1 we know that nodes of degree 2 must have physical links to nodes of degree 4 or higher and from Lemma 2, we know that at most one degree 2 node can have a physical link to each node of degree 4 or degree 5 , therefore

$$
d_{2} \square \frac{d_{4}}{2}+\frac{d_{5}}{2}+\square_{i=6}^{N \square 1} \frac{i}{2} d_{i} .
$$

Substituting Eq. (8) into Eq. (7) yields

$$
L \geq 2 d_{2}+\frac{3}{2} d_{3}+\frac{3}{2} d_{4}+2 d_{5} \text {. }
$$

Since nodes of degree $i$ add a minimum of $i / 2$ physical links to the physical topology,

$$
\begin{aligned}
L & \geq \frac{2 d_{2}+3 d_{3}+4 d_{4}+5 d_{5}+6\left(N \square d_{2} \square d_{3} \square d_{4} \square d_{5}\right)}{2} \\
& =3 N \square 2 d_{2} \square \frac{3}{2} d_{3} \square d_{4} \square \frac{3}{2} d_{5}
\end{aligned}
$$

Equations (9) and (10) require that the number of physical links must be

$$
L \geq \max \left(2 d_{2}+\frac{3 d_{3}}{2}+\frac{3 d_{4}}{2}+2 d_{5}, 3 N \square 2 d_{2} \square \frac{3 d_{3}}{2} \square d_{4} \square \frac{3 d_{5}}{2}\right) \text {. }
$$

We can determine the values of $d_{2}, d_{3}, d_{4}$, and $d_{5}$ that minimize the number of physical links required. The minimum value occurs when,

$$
\begin{aligned}
& 2 d_{2}+\frac{3}{2} d_{3}+\frac{3}{2} d_{4}+2 d_{5}=3 N \square 2 d_{2} \square \frac{3}{2} d_{3} \square d_{4} \square \frac{3}{2} d_{5}, \text { i.e., } \\
& \text { when } 2 d_{2}=\frac{3 N \square 3 d_{3} \square \frac{5}{2} d_{4} \square \frac{5}{2} d_{5}}{2} . \text { Substituting this }
\end{aligned}
$$

value of $2 d_{2}$ into Equation (9) yields

$$
L \geq \frac{3 N}{2}+\frac{d_{4}}{4}+\frac{3 d_{5}}{4} \geq \frac{3 N}{2} \text {. }
$$

QED.

To prove Theorem 4, we first develop the following three lemmas that are derived from Theorem 1.

Lemma 3: A node of degree 6 or degree 7 can have physical links to at most two nodes of degree 2 .

Proof: Suppose three nodes of degree 2 have physical links to a single degree 7 node. Then the cut set consisting of the three degree 2 nodes and the degree 7 node contains only seven links, which is less than the 8 links required by Theorem 1.

Lemma 4: A degree 3 node can have a physical link to at most one other degree 3 nodes.

Proof: Suppose a node of degree 3 has physical links to two other nodes of degree 3 . Then the cut set consisting of the three degree 3 nodes contains only five links, which is less than the 6 links required by Theorem 1 .

Lemma 5: A degree 4 node can have physical links to at most two degree 3 nodes.

Proof: Suppose a node of degree 4 has physical links to three nodes of degree 3 . Then the cut set consisting of the three degree 3 nodes and the degree 4 node contains only seven links, which is less than the 8 links required by Theorem 1. 
Proof of Theorem 4: Let $d_{i}$ be the number of nodes with degree $i$ in the physical topology. Then the number of links in the physical topology, $L$, is

$$
L=d_{2}+\frac{3 d_{3}}{2}+2 d_{4}+\frac{5 d_{5}}{2}+3 d_{6}+\frac{7 d_{7}}{2}+\prod_{i=8}^{N \square 1} \frac{i d_{i}}{2}
$$

From Lemmas 1, 2, and 3, we get the following restriction on the number of degree 2 nodes:

$$
d_{2} \square \frac{d_{4}}{2}+\frac{d_{5}}{2}+d_{6}+d_{7}+\prod_{i=8}^{N \square 1} \frac{i}{2} d_{i} .
$$

Substituting Eq. (14) into Eq. (13) yields

$$
L \geq 2 d_{2}+\frac{3}{2} d_{3}+\frac{3}{2} d_{4}+2 d_{5}+2 d_{6}+\frac{5}{2} d_{7} \text {. }
$$

Lemmas 4 and 5 yield the following restriction on the number of degree 3 nodes:

$$
3 d_{3} \square d_{3}+2 d_{4}+\square_{i=5}^{N \square 1} i d_{i} .
$$

Substituting Eq. (16) into Eq. (13) yields

$$
L \geq d_{2}+\frac{5}{2} d_{3}+d_{4} \text {. }
$$

Since nodes of degree $i$ add a minimum of $i / 2$ physical links to the physical topology,

$$
\begin{gathered}
L \geq \frac{\prod_{i=2}^{7} i d_{i}+8\left(N \square d_{2} \square d_{3} \square d_{4} \square d_{5} \square d_{6} \square d_{7}\right)}{2} . \\
=4 N \square 3 d_{2} \square \frac{5}{2} d_{3} \square 2 d_{4} \square \frac{3}{2} d_{5} \square d_{6} \square \frac{1}{2} d_{7}
\end{gathered}
$$

Equations (15), (17), and (18) require that the number of physical links must be

$$
\begin{aligned}
& L \geq \max \left(2 d_{2}+\frac{3 d_{3}}{2}+\frac{3 d_{4}}{2}+2 d_{5}+2 d_{6}+\frac{5 d_{7}}{2}, d_{2}+\frac{5 d_{3}}{2}\right. \\
& \left.+d_{4}, 4 N \backslash 3 d_{2} \square \frac{5 d_{3}}{2} \square 2 d_{4} \square \frac{3 d_{5}}{2} \square d_{6} \square \frac{d_{7}}{2}\right)
\end{aligned}
$$

We minimize the right hand side of Equation (19) using a linear program to find that $L \geq 1.5 \overline{5} \mathrm{~N}$ for all values of $N$. QED.

To prove Theorem 5 we show that for any physical topology with fewer than $2(N \square 2)$ links, we can find an $N \square 2$ node ring logical topology where each logical link requires two physical links (for a total of 2(NQ2) links). Hence a physical topology with fewer than $2(N \square 2)$ links cannot support all $N \square 2$ node logical topologies. The proof of Theorem 5 uses Lemma 6 below.

Lemma 6: Consider an $N$ node physical topology with $\square 2 N \square 5$ physical links. Let $d_{i}$ be the degree of the $i^{\text {th }}$ largest degree node in the $N$ node topology and assume each node has a minimum degree of two. Then $d_{i} \square \frac{2 N \square 10+2 i}{i}$.
Proof: Since there are at most $2 N \square 5$ physical links in the physical topology, the sum of the degrees of all the nodes must be less than or equal to $4 N \square 10$. Equivalently,

$$
\prod_{k=1}^{i \square 1} d_{k}+d_{i}+\underset{k=i+1}{\prod_{k}^{N}} d_{k} \square 4 N \square 10 \text {. }
$$

To determine an upper bound on $d_{i}$, move the sums on the left hand side of Equation (20) to the right hand side. Since the degree of each node is at least two,

$$
\begin{aligned}
\prod_{k=i+1}^{N} d_{k} & \geq 2(N \square i), \text { thus } \\
d_{i} & \square 4 N \square 10 \square 2(N \square i) \square \prod_{k=1}^{i \square 1} d_{k} \\
& =2 N \square 10+2 i \square \prod_{k=1}^{i \square 1} d_{k} .
\end{aligned}
$$

Since $d_{i}$ is the degree of the $i^{\text {th }}$ largest degree node, we know that its degree must be less than the average of the of the larger degree nodes, i.e., $d_{i} \square \frac{\sum_{k=1}^{i \square 1} d_{k}}{i \square 1}$. Equivalently,

$$
\prod_{k=1}^{i \square 1} d_{k} \geq d_{i}(i \square 1) .
$$

Substituting Equation (22) into Equation (21) yields,

$$
d_{i} \square \frac{2 N \square 10+2 i}{i} \text {. }
$$

Proof of Theorem 5: The proof is by construction of an $N \square 2$ node logical topology that requires at least $2 N \square 4$ physical links. The proof is divided into four cases corresponding to physical topology size. In the first case, we show that the Theorem holds for all rings of size greater than or equal to 12 . The other three cases establish the proof for rings of size less than 12 .

In Case 1, we consider physical topologies of size $N \geq 12$. We start by removing the two largest degree nodes from the physical topology. The resulting physical topology, $P$, has $m=N \square 2$ nodes, with degrees $d_{3}$ through $d_{N}$. We show below that there exists a logical ring topology that traverses these $N \square 2$ nodes and requires more than $2 N \square 5$ physical links.

Consider the inverse of the $m$ node physical topology, denoted $\widehat{P}$, where if link $(i, j) \square P$, then $(i, j) \square \widehat{P}$ and if $(i, j) \square P$, then $(i, j) \square \widehat{P}$. We show below that there exists a Hamiltonian cycle in this inverse graph. The existence of a Hamiltonian cycle in the inverse graph implies that there exists a sequence of $N \square 2$ nodes with direct physical links connecting them in the inverse topology. Hence, these $N \square 2$ nodes cannot be connected using direct physical links in the original physical topology, thus each logical link must utilize at least two physical links in the original physical topology. Thus the logical ring corresponding to the Hamiltonian cycle in the inverse graph requires a 
minimum of $2 N \square 4$ physical links in the original topology. Next, we must prove our claim that a Hamiltonian cycle exists in the inverse topology. Let $i$ be the index of the $i^{\text {th }}$ largest degree node in the original topology and let $\bar{d}_{i}$ denote its degree in the inverse graph $\widehat{P}$. Since $\widehat{P}$ has $N \square 2$ nodes, $\hat{d}_{i} \geq N \square 3 \square d_{i}$. If some of the links of the $i^{\text {th }}$ largest degree node in the original topology were connected to nodes $i=1$ and $i=2$, then the degree of node $\hat{d}_{i}$ is strictly greater than $N \square 3 \square d_{i}$. Thus $\hat{d}_{i+1}$ can be less than $\hat{d}_{i}$. However, the degree of the smallest degree node in $\widehat{P}$ is always $\geq N \square 3 \square d_{3}$ and the degree of the next smallest degree node is always $\geq N \square 3 \square d_{4}$, etc. Let $e_{j}$ denote the degree of the $j^{\text {th }}$ smallest degree node in $\hat{P}$. Then $e_{j} \geq N \square 3 \square d_{j+2}$.

Before we can proceed we must cite the following Lemma which provides a sufficient condition for the existence of a Hamiltonian cycle in a graph.

Lemma 7 [11, p.350]: Given a graph with $m$ nodes with degrees $e_{1} \square e_{2} \ldots \square e_{m}$. If $e_{j}>j$ for $1 \square j<m / 2$, the graph contains a Hamiltonian cycle.

Applying the above Lemma to the inverse graph, the inverse graph $\hat{P}$ contains a Hamiltonian cycle if the degree of the nodes in the inverse graph are such that $e_{j}>j$ for

$1 \square j<m / 2$. Rewriting the requirement for a Hamiltonian cycle in terms of $d_{i}$ and $N$ yields,

$$
e_{i \square 2} \geq N \square 3 \square d_{i}>i \square 2 \text { for } 3 \square i<\frac{N}{2}+1 \text {. }
$$

Using Equation (23) as an upper bound for $d_{i}$ in Equation (24), with some algebraic manipulation yields the following requirement:

$$
(N \square i \square 3) i \square 2 N+10>0 \text { for } 3 \square i<\frac{N}{2}+1 .
$$

The roots of the left hand side of equation (25) occur at $i=2$ and $i=N \square 5$. Hence, Equation (25) is true if $i>2$ and $i<N \square 5$. Thus, Equation (24) is satisfied if $\frac{N}{2}+1 \square N \square 5$. Consequently, there exists a Hamiltonian cycle in the inverse graph $\widehat{P}$ for all $N \geq 12$. The resulting Hamiltonian cycle corresponds to a logical topology that requires for each logical link a minimum of two physical links in the original physical topology, i.e., this logical topology cannot be embedded in the original physical topology with fewer than $2 N \square 4$ physical links.

For case 2, we consider physical topologies of size $N=6$ and $N=7$. Recall from Theorem 2 that a minimum of $4 N / 3$ physical links are required to support rings of size 4. Since $2 N \square 5<4 N / 3$ for $N \square 7,4$ node rings cannot be supported, thus Theorem 5 holds. Similarly, case 3 corresponds to physical topologies of size $N=8$ and $N=9$. From Theorem 3, a minimum of $3 N / 2$ physical links are needed to support rings of size 6 . Again, $2 N \square 5<3 N / 2$ for $N \square 9$ and Theorem 5 still holds. Finally, case 4 corresponds to physical topologies of size $N=10$ and $N=11$. Theorem 4 requires a minimum of $1.5 \overline{5} \mathrm{~N}$ physical links to support rings of size 8 . Since $2 N \square 5<1.5 \overline{5} N$ for $N \square 11$, Theorem 5 still holds and $2 N \Pi 5<1.5 \overline{5} N$ physical links are insufficient to support rings of size $N \square 2$ for all $N \sqcap 11$.

\section{References:}

[1] S. Ramamuthy and B. Mukherjee, "Survivable WDM Mesh Networks: Part I - Protection," Infocom '99, New York, March, 1999.

[2] S. Ramamuthy and B. Mukherjee, "Survivable WDM Mesh Networks: Part II -Restoration," ICC '99, Vancouver, CA, June, 1999.

[3] O. Gerstel, R. Ramaswami, and G. Sasaki, "Fault Tolerant Multiwavelength Optical Rings with Limited Wavelength Conversion,” Infocom '97, Kobe, Japan, April 1997.

[4] M. Medard, S. Finn and R. A. Barry, "WDM Loopback Recovery in Mesh Networks," Infocom '99, New York, March, 1999.

[5] A. Fumagalli, et. al., "Survivable Networks Based on Optimal Routing and WDM Self-Healing Rings," Infocom '99, New York, March, 1999.

[6] O. Crochat and J.Y. Le Boudec, "Design Protection for WDM Optical Networks," IEEE JSAC, Vol. 16, No. 7, Sept. 1998.

[7] B.T. Doshi, S. Dravida, P. Harshavardhana, O. Hauser, and Y. Wang, "Optical Network Design and Restoration," Bell Labs Technical Journal, January-March 1999.

[8] M. Kodialam and T.V. Lakshman, "Minimum Interference Routing with Application to MPLS Traffic Engineering," Infocom, 2000, Tel Aviv, Israel, April, 2000.

[9] E. Modiano and A. Narula-Tam, "Survivable Routing of Logical Topologies in WDM Networks," Infocom 2001, Anchorage, AK, April 2001.

[10] E. Modiano and A.Narula-Tam, "Survivable lightpath routing: a new approach to the design of WDM-based networks," IEEE Journal of Selected Areas in Communication, May 2002.

[11] M.Gondran and M. Minoux, Graphs and Algorithms, John Wiley and Sons, 1984.

[12] A. Narula-Tam and E. Modiano, "Load Balancing Algorithms for WDM-based IP networks," Infocom, 2000, Tel Aviv, Israel, April, 2000.

[13] A. Narula-Tam and E. Modiano, "Dynamic Load Balancing in WDM Packet Networks with and without Wavelength Constraints," IEEE Journal of Selected Areas in Communications, October 2000. 